\title{
The development of intruder attack in colonies of laboratory rats
}

\author{
ROBERT J. BLANCHARD, LOREY K. TAKAHASHI, and D. CAROLINE BLANCHARD \\ University of Hawaii, Honolulu, Hawaii 96822
}

\begin{abstract}
Most attacks by rat colony members on strange intruders are made by a single dominant male. Such dominance, and the attack behaviors themselves, develop in a relatively fixed sequence over sessions with strange intruders. The entire sequence of attack on intruders occurs earlier in the intruder sessions for older rat colonies than in those for newly established colonies: conversely, more attack is seen in colonies with prior intruder experience than for intruder-naive colonies of equivalent age. Thus both experience within the colony and specific experience with strange intruders influence the rate of development of attack on intruders by dominant colony rats.
\end{abstract}

Barnett has described $(1960,1975)$ a profound discrepancy between the reactions of wild rat colonies and those of laboratory rat colonies to a conspecific intruder: the wild rats attack and injure or kill the intruder, while albino rats do not. This difference has been a major contributor to the view that laboratory rats have been so altered in the process of domestication as to make them an unrepresentative and atypical population for the study of such traits as aggression.

However, recent studies of albino rat colonies have produced consistent and dramatic attacks on introduced conspecifics (Blanchard, Fukunaga, Blanchard, \& Kelley, 1975; Luciano \& Lore, 1975). These colonies have produced wounding and mortality rates for intruders which are very much equivalent to those shown in wild rat colonies under similar circumstances (Blanchard et al., 1975). Analysis of the specific agonistic behaviors shown in the colonies by dominant colony rats and by the intruders suggest a clear separation of attacker and defender reactions (Blanchard \& Blanchard, in press). This attack and defense analysis has been applied to the reactions seen in other commonly used "aggression" tasks and has been found to fit these behaviors as well (Blanchard \& Blanchard, in press; Blanchard, Blanchard, \& Takahashi, in press). Finally, the specific behaviors seen in albino rat attack and defense appear to be very similar to those seen in wild rats (Blanchard \& Blanchard, in press).

In view of the apparent generality of the attack and defense analysis based on albino rat colonies, it is important to determine why these colonies show attack while earlier laboratory rat colony studies did not. Since the earlier, nonattacking albino rats had had a shorter period of colony experience and fewer intruders than the colonies in which attack was seen, these factors are examined in the present experiments. These experiments also provide a systematic description of the development of intruder-attack behaviors among members of labora- tory rat colonies and of the development of a dominant male for each colony.

\section{METHOD AND PROCEDURE}

\section{Subjects}

Each of the 16 rat colonies used consisted of three male and three female albino rats, ranging in age from 115 to 140 days with a mean of 131 days at the time of colony formation. In addition, 216 male rats of equivalent age and weight were used as intruders. All of these rats had been bred in the animal colony of the University of Hawaii Department of Psychology. They were derived from Wistar stock supplied by Simonsen Laboratories. Both the colony animals and the intruders had been born in standard laboratory litter cages, with one female and her litter in each cage, and had been removed from these cages when weaned at about 30 days of age. Between weaning and placement in the colony or use as intruders, these animals were singly housed in standard laboratory cages under a standard laboratory maintenance schedule with food and water freely available.

\section{Apparatus}

The colonies were each housed in a $120 \times 60 \times 90 \mathrm{~cm}$ plywood compartment with an outer covering of $2 \times 2$ wire mesh. The tile flooring of the colonies was covered by wood shavings; food and water were freely available at all times.

\section{Experimental Procedure}

Half of the colonies, randomly selected, were designated "early-introduction colonies." while the remainder were "lateintroduction colonies." For the early introduction colonies, strange rats were introduced beginning 1 week after the colony was established. One intruder was introduced each week for a total of 18 weeks. The late-introduction colonies were given the first intruders during Week 10 after colony formation. For these colonies, intruders were presented once per week until Week 18 after colony formation.

During the 10 -min intruder trials, two trained observers monitored the behavior of the colony males. The following behaviors were observed and measured: Latency to piloerect-time until clear erection of back hairs on the observed animal. Lateral attack (duration)-observed animal moves lateral to and within $5 \mathrm{~cm}$ of the intruder, frequently with arched back and lowered head. On-top-of (duration)-observed animal is standing over the intruder, which is lying on its back. Boxing (duration)-observed 
animal is in an upright boxing posture with forepaws off the floor, oriented toward and within $5 \mathrm{~cm}$ of the intruder. Contact (duration)-observed animal is within $3 \mathrm{~cm}$ of the intruder, but not lateral to, or boxing with, the intruder. The number of bites observed to be made by each colony animal on the intruder were also counted, and, at the conclusion of each test session, the number and location of bite wounds penetrating the skin of the intruder were examined and recorded.

\section{RESULTS}

\section{Differential Attack by Alpha Males}

Figure 1 presents the number of bites by each colony male at each intruder. As this figure indicates, regardless of the time of intruder introduction (early vs. late colonies), a single male in each colony soon came to make a clear preponderance of bites on intruders. As in previous studies of colony attack (cf. Blanchard et al., 1975; Blanchard, Blanchard, Takahashi, \& Kelley, in press) the designation of one animal as the dominant or alpha male of the colony was based on a single attack measure, biting.

Table 1 presents measures reflecting the proportions of attack behaviors for each colony that can be attributed to the alpha male of those colonies. This table makes it clear that biting was by no means distributed randomly among the three males of each colony. Even during the early weeks of intruder introduction for the early-introduction colonies, the animal designated as the

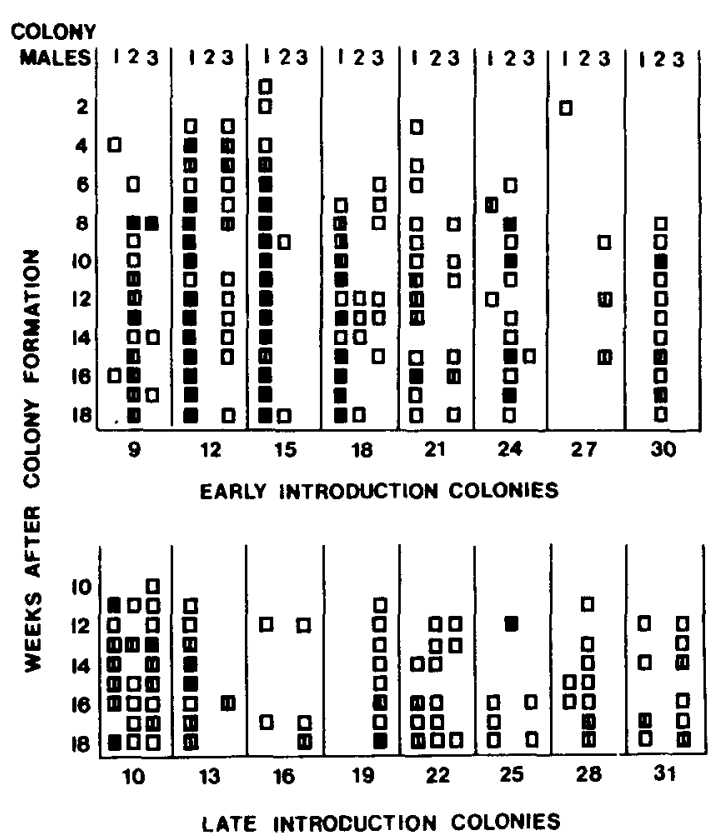

ATTACK BY EACH COLONY MALE ON INTRODUCED STRANGERS: $\begin{array}{lll}\text { LIGHT; } & 1-5 \text { BITES } \\ \text { MEDIUM; } 6-10 " & " \\ \text { HEAVY: }>10 " & \square\end{array}$

Figure 1. Attack by each male rat (three per colony) of colonies with early vs. late introduction of intruders, on the introduced strange rats. Each weekly test session was $10 \mathrm{~min}$ in length and involved a single intruder.
Table 1

Attack Behaviors of Alpha and Other Colony Males of Colonies With Early or Late Introduction of Intruders

\begin{tabular}{|c|c|c|c|c|c|c|}
\hline \multirow{4}{*}{$\begin{array}{c}\text { Attack } \\
\text { Behavior }\end{array}$} & \multirow{2}{*}{\multicolumn{3}{|c|}{$\begin{array}{l}\begin{array}{c}\text { Percentage of Tests } \\
\text { With Alpha Showing } \\
\text { Most Attack Behavior }\end{array} \\
\text { Colonies }\end{array}$}} & \multicolumn{3}{|c|}{$\begin{array}{c}\text { Alpha Behavior as a } \\
\text { Percentage of Total } \\
\text { Attack Behavior }\end{array}$} \\
\hline & & & & \multicolumn{3}{|c|}{ Colonies } \\
\hline & \multicolumn{2}{|c|}{ Early } & \multirow{2}{*}{$\frac{\text { Late }}{10-18}$} & \multicolumn{2}{|c|}{ Early } & \multirow{2}{*}{$\frac{\text { Late }}{10-18}$} \\
\hline & $1-9 *$ & $10-18$ & & $1-9$ & $10-18$ & \\
\hline Piloerection & 65.50 & 82.63 & 56.50 & & & \\
\hline Lateral Attack & 75.93 & 87.24 & 79.31 & 84.96 & 90.28 & 81.36 \\
\hline On-Top-of & 65.62 & 85.26 & 71.87 & 84.80 & 88.30 & 78.08 \\
\hline Boxing & 45.89 & 65.66 & 54.28 & 34.23 & 64.20 & 51.04 \\
\hline Contact Time & 61.81 & 88.89 & 64.58 & 49.42 & 64.95 & 47.36 \\
\hline Bites & 73.92 & 95.66 & 85.42 & 82.39 & 91.82 & 72.22 \\
\hline
\end{tabular}

*Weeks

alpha made the most bites on the intruder during more than $70 \%$ of the tests and also accounted for over $80 \%$ of all bites on intruders. During Weeks 10-18, the rats designated as alphas always made the most bites on intruders in five of the eight early introduction colonies, while in the remaining three colonies alpha showed more biting in eight of the nine test sessions. Chi-square tests on the summed bite data indicated that biting was not randomly distributed among the males of any colony $\left[\chi^{2}(2)=18\right.$, or 12.66 for all early-introduction colonies, $\mathrm{p}<.01$ for each colony]. In the late-introduction colonies, dominance was also clear, with a single animal showing more biting on over $85 \%$ of the test sessions in which biting was observed. Statistically reliable nonrandom bite distributions were obtained in five of the eight late-introduction colonies $\left[\chi^{2}(2)\right.$ $=18$, or $12.66, p<.01]$, while the remaining three colonies approached, but did not reach, an acceptable level of statistical significance $\left[x^{2}(2)=3.53,3.33\right.$, and $3.62, \mathrm{p}>.05]$.

The differences in biting dominance were paralleled by clear differences in speed of piloerection and duration of lateral attack and on-top-of behaviors. In each case, the rat designated as alpha tended to show more of each of these behaviors than any other colony member. On Trials $10-18$ for the late colonies, alpha males piloerected earlier and showed more lateral attack and on-top-of behaviors during more than $80 \%$ of all tests and accounted for approximately $90 \%$ of these two behaviors (duration of piloerection was not measured). Boxing was somewhat less clearly restricted to the dominant animal, a feature which is of considerable interest in terms of analyses suggesting that boxing in the alpha is more an action imposed by vibrissae-contact with the intruder than a genuine attack strategy (Blanchard \& Blanchard, in press). Thus, the present findings are consonant with previous suggestions that lateral attack, on-top-of, and biting are the clearest attack behaviors of colony rats, while boxing is a more equivocal response (Blanchard \& Blanchard, in press). The major conclusion to be derived from these data, 
however, is that the early weeks of colony experience appear to produce dominance of a single animal, in terms of attacks made on strangers introduced into the colony. A similar conclusion was reached by Barnett (1975) in his studies of wild rat colonies. Such dominance in attacks on intruders does not necessarily involve dominance within the colony, although single-sex groups of rats do establish a within-colony dominance system (Grant \& Chance, 1958), nor does it imply dominance in food-getting, such as predatory attacks on frogs (DeSisto \& Huston, 1970).

\section{Development of Attack in Early-vs. Late-Introduction Colonies}

Since attack by animals other than the colony alpha was infrequent and sporadic for all colonies, the data to be presented on the development of attack involve primarily the behaviors of these alpha males. Table 2 presents the mean intruder test session in which each attack behavior of the alpha rat first appeared. As this table indicates, there was a rather consistent order of appearance of individual alpha behaviors for both earlyand late-introduction colonies: contact with the intruder was the first alpha behavior to appear, occurring for all colonies in the first intruder session. Several sessions later, usually within the same adjacent sessions, piloerection, lateral attack, and biting by the alpha male were seen. On-top-of behaviors did not occur until later for the early introduction colonies, although they tended to occur in the same test session as biting for the late-introduction colonies. In terms of evidence indicating that boxing is not a typical alpha attack behavior, it is interesting to note that the appearance of boxing for the alpha male was quite variable for early vs. late colonies, being the first behavior (after contact) to occur in the early colonies and the last to be seen in the late colonies. It is also interesting to note that contact with the intruders, early in the intruder experience of each colony, was poorly correlated with later attack behavior for individual animals: before any biting was seen in the colony, males that were later to be designated as alphas accounted for $38.75 \%$ of contact times, which was not significantly different from the $33.33 \%$ expected by chance.

The alpha males of the late-introduction colonies,
Table 2

Test Week in Which Each Attack Behavior was First Observed for Early vs. Late Colonies

\begin{tabular}{lcc} 
Attack Behavior & $\begin{array}{c}\text { Early } \\
\text { Colonies }\end{array}$ & $\begin{array}{c}\text { Late } \\
\text { Colonies }\end{array}$ \\
\hline Piloerection & 5.37 & 2.50 \\
Lateral Attack & 5.75 & 2.87 \\
On-Top-of & 7.12 & 3.25 \\
Boxing & 4.37 & 4.25 \\
Contact & 1.00 & 1.00 \\
Biting & 5.87 & 3.25 \\
\hline
\end{tabular}

which had had 10 weeks of colony experience before any intruders were provided, showed a consistent tendency to attack the strange rats earlier in the intruder series. The principal alpha male attack pattern behaviors, piloerection $[\mathrm{t}(14)=3.40, \mathrm{p}<.01]$, lateral attack $[\mathrm{t}(14)=2.83, \mathrm{p}<.05]$, on-top-of $[\mathrm{t}(14)=2.74, \mathrm{p}<$ $.05]$, and boxing $[\mathrm{t}(14)=2.19, \mathrm{p}<.05]$ all appeared reliably earlier for the late-introduction colonies than for the colonies in which strangers were presented soon after colony formation. Neither alpha contact nor boxing differentiated the early vs. late colonies, providing another area of evidence that these behaviors are not essential components of the alpha attack pattern.

The latency, duration, and incidence data for the alpha attack behaviors show a very similar pattern. Table 3 presents these data. In terms of changes in attack patterns from early intruder trials to late intruder trials (for the early-introduction colonies), the latency to piloerect decreases reliably $[\mathrm{t}(7)=8.27, \mathrm{p}<.001]$, while the duration of on-top-of $[\mathrm{t}(7)=3.76, \mathrm{p}<.01]$, contact $[\mathrm{t}(7)=2.87, \mathrm{p}<.05]$, and boxing $[\mathrm{t}(7)=$ $3.28, \mathrm{p}<.01]$ were all greater during Trials $10-18$ than during Trials 1-9. The increase in the duration of the lateral attack behavior approached, but did not reach, an acceptable level of statistical significance $[\mathrm{t}(7)=2.20$, $p=.06]$. The number of bites increased significantly during the later half of the test sessions $[\mathrm{t}(7)=4.22$, $p<.01]$ for the early-introduction colonies.

Comparison of the first three intruder test sessions for early- vs. late-introduction colonies (Weeks $1-3$ for the early colonies and Weeks 10-12 for the late colonies) indicated that the late colonies showed more rapid piloerection $[\mathrm{U}(8,8)=0, \mathrm{p}<.001]$ and reliably more on-

Table 3

Development of Attack Behaviors to Intruders by Male Rats in Colonies With Early or Late Intruders

\begin{tabular}{|c|c|c|c|c|c|c|c|c|c|}
\hline \multirow[b]{2}{*}{ Behavior } & \multicolumn{6}{|c|}{ Early Colonies (Weeks) } & \multicolumn{3}{|c|}{ Late Colonies (Weeks) } \\
\hline & $1-3$ & 4-6 & $7-9$ & $10-12$ & $13-16$ & $17-19$ & $10-12$ & $13-16$ & $17-19$ \\
\hline Piloerection & 600.00 & 402.50 & 241.87 & 75.41 & 48.33 & 54.12 & 340.62 & 159.16 & 104.54 \\
\hline Lateral Attack & 4.75 & 8.87 & 15.83 & 11.75 & 41.58 & 45.37 & 5.66 & 24.29 & 29.00 \\
\hline On-Top-of & .33 & 4.37 & 11.79 & 19.00 & 13.33 & 48.50 & 11.54 & 8.58 & 50.83 \\
\hline Boxing & 1.87 & 2.08 & 5.04 & 3.00 & 16.00 & 11.79 & .50 & 12.04 & 10.41 \\
\hline Contact Time & 178.00 & 207.00 & 260.00 & 228.25 & 312.04 & 329.76 & 189.54 & 247.37 & 309.87 \\
\hline Bites & .33 & 2.42 & 6.92 & 13.00 & 9.38 & 9.45 & 1.33 & 4.58 & 5.66 \\
\hline Lesions & .08 & 1.92 & 4.42 & 6.54 & 6.92 & 10.75 & .75 & 3.37 & 5.96 \\
\hline
\end{tabular}


top-of $[\mathrm{U}(8,8)=10, \mathrm{p}<.01]$ and biting $[\mathrm{U}(8,8)=15$, $\mathrm{p}<.05$ ] behaviors. Lateral attack differences $(U=18)$ were in the same direction, but failed to reach adequate levels of statistical significance for the two groups during these early trials. Boxing and contact times were clearly not different for the two groups. Thus, the major indices of attack were developed earlier in the colonies which had been established for a considerable period before the first strangers were introduced. This finding is congruent with Luciano and Lore's (1975) report of more attacks on intruders by colony rats raised in groups rather than in isolation.

Comparison of attack tendencies for the early- and late-introduction colonies at the same point after colony formation (Weeks 10-12 for both groups) demonstrates that experience with intruders was also important in the development of attack behavior. The early-introduction colonies, which had had 9 weeks of prior experience with intruders, showed reliably faster piloerection [ $\mathrm{t}(14)$ $=4.44, \mathrm{p}<.001]$, more boxing $[\mathrm{t}(14)=2.30, \mathrm{p}<.05]$, and more biting $[\mathrm{t}(14)=2.48, \mathrm{p}<.05]$ than the late colonies (for which these were the first experiences with strange intruders). Differences in lateral attack were again in the same direction as the other attack-measure differences, but were not significant $(t=1.08)$.

In previous studies of attack by colony males on strangers, Blanchard et al. (1975) reported that attack appears to be directed to target sites on the back of the intruder. The present locations of lesions made on the intruders are fully congruent with this finding: $82.75 \%$ of lesions made by the early-introduction colonies and $80.00 \%$ of those made by the late-introduction colonies occurred on the backs of the intruders. Head lesions accounted for $6.33 \%$ and $4.62 \%$, limb lesions were $6.60 \%$ and $11.15 \%$, and thorax and abdomen lesions were $3.91 \%$ and $3.85 \%$, respectively, for intruders into the early or late colonies. These proportions are obviously compatible with an analysis suggesting that attack of alpha male rats is directed toward the back and away from the ventral surfaces of conspecifics (Blanchard et al., in press), while intruder behavior serves as an effective back defense.

\section{DISCUSSION}

It has long been clear that wild, pigmented rats show a higher level of attack behaviors than do albino laboratory rats (Barnett, 1960), especially when the wild rats have been reared in a "natural" environment (Barnett \& Stoddart, 1969). In the general circumstances in which rats have evolved, rats tend to be relatively colonial (Telle, 1966) and thus have frequent experience with familiar conspecific males and females and with strange conspecifics. In indicating that experience within a mixed-sex colony plus occasional exposure to strange conspecific males can also produce a high level of attack in laboratory rats, the present study indicates that common factors are important in the control of ag- gressive tendencies of wild and laboratory rat species. The specific attack behaviors of wild and laboratory males appear to be quite similar, suggesting that the colony situation produces an agonistic behavior pattern that is very typical of the species, although it is quite different from the behaviors seen in such tasks as "reflexive fighting" which have often been used as a major measure of aggression in the laboratory rat (Blanchard, Blanchard, \& Takahashi, in press).

The present results provide several items of information relevant to the mechanism by which conspecific experience facilitates attack in rats. First, both withincolony experience and experience of strangers appear to be very important in the development of intruder attack. It seems obvious that an opportunity to fight, and to experience victory, might be a common factor for both types of conspecific experience. In this context, the experience of victory could therefore serve as a reward for fighting, and the specific behaviors preceeding such victory might be regarded as operants, learned on the basis of their value in producing this outcome. In fact, Scott has demonstrated (1966) that experience of victory does facilitate subsequent attack in the mouse.

It should be noted, however, that even though victory may facilitate fighting in male rats, the reward afforded by victory is probably intrinsic rather than based on external rewards associated with such victory. In the present situation, food, water, space, and access to females were all abundant and appeared to be freely utilized. Although the dominant male of each colony may conceivably have derived some relatively minor sexual or space utilization advantage from its status as dominant rat, it nevertheless seems extremely unlikely that immediate access to these items was so clearly a consequence of victory as to provide differential reinforcement for the learning of the behaviors associated with victory.

A related finding was that the specific attack behaviors seen were very similar from one colony to the next. This feature makes it very difficult to regard the form of these behaviors as having been developed completely on the basis of their consequences in achieving victory. It is also interesting that biting, the only behavior likely to inflict any physical damage on the opponent, does not appear until after the appearance of piloerection and the lateral attack. Unless these behaviors somehow produce "victory" when used alone, it is difficult to see how they could have learned in the absence of biting. And if they do produce or contribute to victory, then there is obviously some preprogrammed component to the behavior pattern which constitutes dominance in the rat. Finally, these same components appear to be present in attacks by isolated males on strangers (Adams, 1976), again indicating a topographical component which cannot be regarded as the adventitious product of shaping through victory.

These considerations strongly suggest that, although 
the form of attack behaviors in the rat may be sharpened through experience with fighting, there is also a preprogrammed element to such behavior that is common to both wild and laboratory rats. The present studies also indicate that such factors as housing with male and female conspecifics and occasional exposure to strange male conspecifics, experiences undoubtedly present in the circumstances in which wild rats have evolved, are sufficient for the appearance of a well-developed and consistent pattern of conspecific attack in albino rats.

\section{REFERENCES}

ADAMs, D. B. The relation of scent-marking, olfactory investigation, and specific patterns in the isolation-induced fighting of rats. Behaviour, 1976, 56, 286-297.

BARNETT, S. A. Social behavior among tame rats and among wild-white hybrids. Proceedings of the Zoological Society of London. 1960, 134, 611-662.

Barnett, S. A. The rat: A study in behavior (rev. ed.). Chicago and London: University of Chicago Press, 1975.

BarnetT, S. A., \& Stoddart, R. C. Effects of breeding in captivity on conflict among wild rats. Journal of Mammology, $1969,50,321-325$.

Blanchard, R. J., \& Blanchard, D. C. Aggressive behavior in the rat. Behavioral Biology, in press.
Blanchard, R. J., Fununaga, K. K., Blanchard, D. C., \& KELley, M. J. Conspecific aggression in the laboratory rat. Journal of Comparative and Physiological Psychology, 1975, 89, 1204-1209.

Blanchard, R. J., Blanchard, D. C., Takahashi, T., \& KELLEY, M. J. Attack and defensive behavior in the rat. Animal Behaviour, in press.

BlanchaRd, R. J., Blanchard, D. C., \& Takahashi, L. K Reflexive fighting in the albino rat: Aggressive or defensive behavior? Aggressive Behavior, in press.

DESisto, M. J., \& Huston, J. P. Effect of territory on frog-killing by rats. Journal of Genetic Psychology, 1970, 83, 179-184.

Grant, E. C., \& Chance, M. R. A. Rank order in caged rats. Animal Behaviour, 1958, 6, 183-194.

Luciano, D., \& LoRE, R. Aggression and social experience in domesticated rats. Journal of Comparative and Physiological Psychology, 1975, 88, 917-923.

Scort, J. P. Agonistic behavior of mice and rats: A review. American Zoologist, 1966, 6, 683-701.

Telle, H. J. Beitrag zur Kenntnis der Verhaltensweise von Ratten, vergleichend dargestellt bei, Rattus norvegicus and Rattus rattus. Zeitschrift für Angewandte Zoologie, 1966 53, 129-196. (Available in English as Technical Translation 1608, Translation Section, National Science Library, National Research Council of Canada, Ottawa, Ontario, Canada.)

(Received for publication January 13, 1977; revision accepted May 17, 1977.) 